\title{
Global Hopf bifurcation for two zooplankton-phytoplankton model with two delays
}

\author{
Renxiang Shi ${ }^{1}$ and Wenguo Yang ${ }^{1 *}$
}

\section{"Correspondence:}

yangwenguo@njucm.edu.cn 'Department of Mathematics, Nanjing University of Chinese Medicine, Nanjing, 210023, China

\begin{abstract}
In this paper, we study the global existence of a bifurcating periodic solution for a two zooplankton-phytoplankton model with two delays. First, we demonstrate that the bifurcating periodic solution exists when one delay increases and the other delay remains unchanged. Second, we give simulation to describe the bifurcating periodic solution when one delay increases. Our work answers the question in Sect. 5 (Shi and Yu in Chaos Solitons Fractals 100:62-73, 2017).
\end{abstract}

Keywords: Zooplankton-phytoplankton model; Delays; Limit cycle; Hopf bifurcation

\section{Introduction}

The dynamics of a plankton model is important for an aquatic system: phytoplankton produce oxygen by photosynthesis and absorb nearly half carbon dioxide, so phytoplankton exert great influence on our ecosystem. There have been many works about the dynamics of a phytoplankton-zooplankton model in recent years. As we know, delays always exist in nature, which could bring rich dynamics in a large range of biological systems. So the delayed phytoplankton-zooplankton model has attracted much interest [1-10]; the model contains two delays, that is, gestation delay for zooplankton and the maturity delay for toxic phytoplankton, which have been studied by many researchers $[1,4,6,8-10]$ in recent years. In $[1,9,10]$, the authors took the gestation delay as a parameter, and the Hopf bifurcation was discussed. In $[4,6,8]$, the author took the delay required for the maturation of toxic phytoplankton as a parameter, and the dynamics of system was studied. We know that there always exist more than one delay in the world, so the dynamics of multiple delays should be more real and interesting. The dynamics of more than one delay have turned up in recent years [1, 6, 11-13]. For example, the author [6] discussed Hopf bifurcation of the following phytoplankton-zooplankton system with two delays:

$$
\left\{\begin{array}{l}
\frac{d P_{1}}{d t}=r_{1} P_{1}\left(1-\frac{P_{1}}{K}\right)-\alpha_{1} P_{1} P_{2}-\rho_{1} P_{1} Z \\
\frac{d P_{2}}{d t}=r_{2} P_{2}\left(1-\frac{P_{2}}{K}\right)-\alpha_{2} P_{1} P_{2}-\rho_{2} P_{2} Z, \\
\frac{d Z}{d t}=\left(r_{1} P_{1}+r_{2} P_{2}\right) Z-d Z-\theta_{1} P_{1}\left(t-\tau_{1}\right) Z-\theta_{2} P_{2}\left(t-\tau_{2}\right) Z
\end{array}\right.
$$

\section{Springer}

(c) The Author(s) 2020. This article is licensed under a Creative Commons Attribution 4.0 International License, which permits use, sharing, adaptation, distribution and reproduction in any medium or format, as long as you give appropriate credit to the original author(s) and the source, provide a link to the Creative Commons licence, and indicate if changes were made. The images or other third party material in this article are included in the article's Creative Commons licence, unless indicated otherwise in a credit line to the material. If material is not included in the article's Creative Commons licence and your intended use is not permitted by statutory regulation or exceeds the permitted use, you will need to obtain permission directly from the copyright holder. To view a copy of this licence, visit http://creativecommons.org/licenses/by/4.0/. 
where the delays $\tau_{1}$ and $\tau_{2}$ stand for the time required for maturity of two toxic phytoplankton. Besides we studied the Hopf bifurcation with two delays caused by the gestation of two zooplankton [1]:

$$
\left\{\begin{array}{l}
\frac{d P}{d t}=r P\left(1-\frac{P}{K}\right)-\frac{\mu_{1} P Z_{1}}{\alpha_{1}+P}-\frac{\mu_{2} P Z_{2}}{\alpha_{2}+P}, \\
\frac{d Z_{1}}{d t}=\frac{\beta_{1} P\left(t-\tau_{1}\right) Z_{1}\left(t-\tau_{1}\right)}{\alpha_{1}+P\left(t-\tau_{1}\right)}-\frac{\rho_{1} P(t) Z_{1}}{\alpha_{1}+P(t)}-d_{1} Z_{1}-g_{1} Z_{1}^{2}, \\
\frac{d Z_{2}}{d t}=\frac{\beta_{2} P\left(t-\tau_{2}\right) Z_{2}\left(t-\tau_{2}\right)}{\alpha_{2}+P\left(t-\tau_{2}\right)}-\frac{\rho_{2} P(t) Z_{2}}{\alpha_{2}+P(t)}-d_{2} Z_{2}-g_{2} Z_{2}^{2},
\end{array}\right.
$$

where $P(t), Z_{i}(t)(i=1,2)$ denote the density of phytoplankton and zooplankton respectively, $r$ is the intrinsic growth rate for phytoplankton, $K$ is the environmental carrying capacity of phytoplankton, $\mu_{i}$ denotes the maximum uptake rate for zooplankton. $\frac{P}{\alpha_{i}+P}$ denotes the Holling $\Pi$ functional response which describes the grazing for zooplankton. $\beta_{i}, \rho_{i}$ are the ratio of biomass conversion and the rate of toxic substance produced by per unit biomass of phytoplankton, $d_{i}$ is the death rate, $g_{i}$ denotes the intrinsic competition coefficients, $\alpha_{i}$ is the half saturation constant for Holling $\Pi$ functional response, apparently $\beta_{i}<\mu_{i}$ holds. We know that Wu [14] gave the condition about the global Hopf bifurcation for a delay differential system. There exist much work about the global Hopf bifurcation [5, 7-11, 15-17]. Especially there are many works on a phytoplankton-zooplankton system [5, 7-10]. For example, we take the gestation delay as a parameter, and the global Hopf bifurcation of the following system is discussed [10]:

$$
\left\{\begin{array}{l}
\frac{d P}{d t}=r P\left(1-\frac{P}{K}\right)-\frac{\mu P Z}{\alpha+P}, \\
\frac{d Z}{d t}=\frac{\rho P(t-\tau) Z(t-\tau)}{\alpha+P(t-\tau)}-\frac{\rho P(t) Z}{\alpha+P(t)}-d Z-g Z^{2} .
\end{array}\right.
$$

By now, there have been little work about the global Hopf bifurcation with two delays. In paper [11], the global existence of a bifurcating periodic solution of a neural network model with two delays was studied by limiting $\tau_{1} \in\left(0, \tau_{10}\right)$ and taking $\tau_{2}$ as a bifurcation parameter.

Based on the above works, we shall study the global Hopf bifurcation of system (1.2) with delays, which is the second question given in Sect. 5 [1]. In this paper we should answer this question: if two delays coexists for system (1.2), limiting $\tau_{1} \in\left(0, \tau_{10}\right)$, when $\tau_{2}$ increases, does the periodic solution exist for ever? This paper is organized as follows: in Sect. 2, we give some preliminary work about system (1.2). In Sect. 3, based on [14], we demonstrate the global existence of a periodic solution, then we give some simulation of the periodic solution of system (1.2).

\section{The mathematical model}

Before our discussion, we give the condition for the existence and uniqueness of positive equilibrium.

We know when delays do not exist, $\frac{d z_{i}}{d t}<0$ as $\beta_{i}-\rho_{i}-d_{i}<0$. Hence we assume that $\beta_{i}-\rho_{i}-d_{i}>0$ holds. From [4], we know that, for system (1.2), a positive equilibrium exists when $h_{3}\left(\frac{d_{1} \alpha_{1}}{\beta_{1}-\rho_{1}-d_{1}}\right)>0, h_{3}\left(\frac{d_{2} \alpha_{2}}{\beta_{2}-\rho_{2}-d_{2}}\right)>0$ hold, where

$$
h_{3}(P)=r\left(1-\frac{P}{K}\left(\alpha_{1}+P\right)^{2}\left(\alpha_{2}+P\right)^{2}-\frac{\mu_{1}}{g_{1}}\left(\left(\beta_{1}-\rho_{1}-d_{1}\right) P-d_{1} \alpha_{1}\right)\left(\alpha_{2}+P\right)^{2}\right.
$$




$$
-\frac{\mu_{2}}{g_{2}}\left(\left(\beta_{2}-\rho_{2}-d_{2}\right) P-d_{2} \alpha_{2}\right)\left(\alpha_{1}+P\right)^{2} .
$$

Furthermore, the positive equilibrium is unique when $h_{3}(P)$ is monotone on $\left(P_{0}, P_{1}\right)$, the definition of $P_{0}, P_{1}$ could be found in [4].

In [1], we discussed the dynamics of system (1.2) under six cases (1) $\tau_{1}=\tau_{2}=0$, (2) $\tau_{1}>$ $0, \tau_{2}=0$, (3) $\tau_{1}=0, \tau_{2}>0,(4) \tau_{1}=\tau_{2}=\tau>0,(5) \tau_{1} \in\left(0, \tau_{10}\right), \tau_{2}>0,(6) \tau_{2} \in\left(0, \tau_{20}\right), \tau_{1}>0$. Now we give two results about case (2) and case (5), which is necessary for our work in this paper.

Lemma 2.1 (Theorem 3.1 [1]) For system (1.2), when $\tau_{1}>0, \tau_{2}=0$, if $H_{21}, H_{22}$ hold, the equilibrium $\left(P^{*}, Z_{1}^{*}, Z_{2}^{*}\right)$ is asymptotically stable for $\tau_{1} \in\left[0, \tau_{10}\right)$, Hopf bifurcation occurs when $\tau_{1}=\tau_{10}$.

Lemma 2.2 (Theorem 3.4 [1]) For system (1.2), when $\tau_{1} \in\left(0, \tau_{10}\right)$, if $H_{51}, H_{52}$ hold, the equilibrium $\left(P^{*}, Z_{1}^{*}, Z_{2}^{*}\right)$ is asymptotically stable when $\tau_{2} \in\left(0, \tau_{20}^{*}\right)$, Hopf bifurcation occurs when $\tau_{2}=\tau_{20}^{*}$.

From the description of dynamics of system (1.2) under case (5), we could obtain

$$
\operatorname{Re}\left(\frac{d \lambda}{d \tau_{2}}\right)^{-1}>0,
$$

where

$$
\begin{aligned}
& \tau_{2 k}^{*(j)}=\frac{1}{\omega_{2 k}^{*}} \arccos \left(\frac{R_{52} R_{53}-R_{51} R_{54}}{R_{51}^{2}+R_{52}^{2}}\right)+\frac{2 j \pi}{\omega_{2 k}^{*}}, \\
& \tau_{20}^{*}=\min \tau_{2 k}^{*(0)}, \quad(k=0,1,2, \ldots),
\end{aligned}
$$

the definition of $H_{21}, H_{22}, H_{51}, H_{52}, R_{51}, R_{52}, R_{53}, R_{54}$, and $\omega_{20}^{*}$ could be found in [1].

\section{Global Hopf bifurcation}

In appendix [1], assuming $\tau_{1}<\tau_{20}^{*}$, we give the property of Hopf bifurcation for Lemma 2.2 by the center manifold theorem and normal form [18]. Based on the above fact, we study the global Hopf bifurcation under case (5) of system (1.2).

Let $X=C\left(\left[-\tau_{2}, 0\right], R^{3}\right), u_{t}(\theta)=\left(P(t+\theta), Z_{1}(t+\theta), Z_{2}(t+\theta)\right), t \geq 0, \theta \in\left[-\tau_{2}, 0\right]$, then system (1.2) becomes

$$
\begin{aligned}
& \dot{u}_{t}=F\left(u_{t}, \tau_{2}, T\right), \\
& F\left(\Phi, \tau_{2}, T\right)=\left(\begin{array}{c}
r \phi_{1}(0)\left(1-\frac{\phi_{1}(0)}{K}\right)-\frac{\mu_{1} \phi_{1}(0) \phi_{2}(0)}{\alpha_{1}+\phi_{1}(0)}-\frac{\mu_{2} \phi_{1}(0) \phi_{3}(0)}{\alpha_{2}+\phi_{1}(0)} \\
\frac{\beta_{1} \phi_{1}\left(-\tau_{1}\right) \phi_{2}\left(-\tau_{1}\right)}{\alpha_{1}+\phi_{1}\left(-\tau_{1}\right)}-\frac{\rho_{1} \phi_{1}(0) \phi_{2}(0)}{\alpha_{1}+\phi_{1}(0)}-d_{1} \phi_{2}(0)-g_{1} \phi_{2}^{2}(0) \\
\frac{\beta_{2} \phi_{1}\left(-\tau_{2}\right) \phi_{(}\left(-\tau_{2}\right)}{\alpha_{2}+\phi_{1}\left(-\tau_{2}\right)}-\frac{\rho_{2} \phi_{1}(0) \beta_{3}(0)}{\alpha_{2}+\phi_{1}(0)}-d_{2} \phi_{3}(0)-g_{2} \phi_{3}^{2}(0)
\end{array}\right)
\end{aligned}
$$

with $\Phi=\left(\phi_{1}, \phi_{2}, \phi_{3}\right)$ and $F: X \times R_{+} \times R_{+} \rightarrow R_{+}^{3}$ is a mapping, we have a mapping $\hat{F}=$ $\left.F\right|_{R_{+}^{3} \times R_{+} \times R_{+}}$by restricting $F$ to the subspace of a constant function in $X$. If $\hat{F}\left(\hat{u}, \hat{\tau}_{2}, \hat{T}\right)=0$, 
then $\left(\hat{u}, \hat{\tau}_{2}, \hat{T}\right)$ is called a stationary point, where

$$
\hat{F}\left(u, \tau_{2}, T\right)=\left(\begin{array}{c}
r P\left(1-\frac{P}{K}\right)-\frac{\mu_{1} P Z_{1}}{\alpha_{1}+P}-\frac{\mu_{2} P Z_{2}}{\alpha_{2}+P} \\
\frac{\beta_{1} P Z_{1}}{\alpha_{1}+P}-\frac{\rho_{1} P Z_{1}}{\alpha_{1}+P}-d_{1} Z_{1}-g_{1} Z_{1}^{2} \\
\frac{\beta_{2} P Z_{2}}{\alpha_{2}+P}-\frac{\rho_{2} P Z_{2}}{\alpha_{2}+P}-d_{2} Z_{2}-g_{2} Z_{2}^{2}
\end{array}\right)
$$

and

$$
D_{u} \hat{F}\left(u, \tau_{2}, T\right)=\left(\begin{array}{lll}
a_{11} & a_{12} & a_{13} \\
a_{21} & a_{22} & a_{23} \\
a_{31} & a_{32} & a_{33}
\end{array}\right)
$$

where

$$
\begin{aligned}
& a_{11}=r-\frac{2 r P}{K}-\frac{\mu_{1} Z_{1}}{\alpha_{1}+P}+\frac{\mu_{1} P Z_{1}}{\left(\alpha_{1}+P\right)^{2}}-\frac{\mu_{2} Z_{2}}{\alpha_{2}+P}+\frac{\mu_{2} P Z_{2}}{\left(\alpha_{2}+P\right)^{2}}, \\
& a_{12}=-\frac{\mu_{1} P}{\alpha_{1}+P}, \quad a_{13}=-\frac{\mu_{2} P}{\alpha_{2}+P}, \\
& a_{21}=\frac{\left(\beta_{1}-\rho_{1}\right) Z_{1}}{\alpha_{1}+P}-\frac{\left(\beta_{1}-\rho_{1}\right) P Z_{1}}{\left(\alpha_{1}+P\right)^{2}}, \quad a_{22}=\frac{\left(\beta_{1}-\rho_{1}\right) P}{\alpha_{1}+P}-d_{1}-2 g_{1} Z_{1}, \quad a_{23}=0, \\
& a_{31}=\frac{\left(\beta_{2}-\rho_{2}\right) Z_{2}}{\alpha_{2}+P}-\frac{\left(\beta_{2}-\rho_{2}\right) P Z_{2}}{\left(\alpha_{2}+P\right)^{2}}, \quad a_{32}=0, \quad a_{33}=\frac{\left(\beta_{2}-\rho_{2}\right) P}{\alpha_{2}+P}-d_{2}-2 g_{2} Z_{2} .
\end{aligned}
$$

$F$ and $\hat{F}$ satisfy the following conditions:

$\left(A_{1}\right) \hat{F} \in C^{2}\left(R_{+}^{3} \times R_{+} \times R_{+}, R_{+}^{3}\right)$,

$\left(A_{2}\right) D_{\hat{u}} \hat{F}\left(\hat{u}, \tau_{2}, T\right)$ is an isomorphism at equilibrium $\left(\hat{u}, \tau_{2}, T\right)$,

$\left(A_{3}\right) F\left(\Phi, \tau_{2}, T\right)$ is differentiable with respect to $\Phi$.

For any stationary solution $\left(\hat{u}, \hat{\tau}_{2}, \hat{T}\right)$, the characteristic matrix of system (1.2) is $\Delta\left(\hat{u}, \tau_{2}\right.$, $T)(\lambda)=\lambda I-D F\left(\hat{u}, \tau_{2}, T\right)\left(e^{\lambda \cdot I}\right)$, that is,

$$
\Delta\left(\hat{u}, \tau_{2}, T\right)(\lambda)=\left(\begin{array}{lll}
b_{11} & b_{12} & b_{13} \\
b_{21} & b_{22} & b_{23} \\
b_{31} & b_{32} & b_{33}
\end{array}\right)
$$

where

$$
\begin{aligned}
& b_{11}=\lambda-r+\frac{2 r \hat{P}}{K}+\frac{\mu_{1} \hat{Z}_{1}}{\alpha_{1}+\hat{P}}-\frac{\mu_{1} \hat{P} \hat{Z}_{1}}{\left(\alpha_{1}+\hat{P}\right)^{2}}+\frac{\mu_{2} \hat{Z}_{2}}{\alpha_{2}+\hat{P}}-\frac{\mu_{2} \hat{P} \hat{Z}_{2}}{\left(\alpha_{2}+\hat{P}\right)^{2}}, \\
& b_{12}=\frac{\mu_{1} \hat{P}}{\alpha_{1}+\hat{P}}, \quad b_{13}=\frac{\mu_{2} \hat{P}}{\alpha_{2}+\hat{P}}, \\
& b_{21}=\frac{\rho_{1} \hat{Z}}{\alpha_{1}+\hat{P}}-\frac{\rho_{1} \hat{P} \hat{Z}_{1}}{\left(\alpha_{1}+\hat{P}\right)^{2}}-\left(\frac{\beta_{1} \hat{Z}_{1}}{\alpha_{1}+\hat{P}}-\frac{\beta_{1} \hat{P} \hat{Z}_{1}}{\left(\alpha_{1}+\hat{P}\right)^{2}}\right) e^{-\lambda \tau_{1}}, \\
& b_{22}=\lambda+d_{1}+2 g_{1} \hat{Z_{1}}+\frac{\rho_{1} \hat{P}}{\alpha_{1}+\hat{P}}-\frac{\beta_{1} \hat{P}}{\alpha_{1}+\hat{P}} e^{-\lambda \tau_{1}}, \quad b_{23}=0 \\
& b_{31}=\frac{\rho_{2} \hat{Z}}{\alpha_{2}+\hat{P}}-\frac{\rho_{2} \hat{P} \hat{Z}_{2}}{\left(\alpha_{2}+\hat{P}\right)^{2}},-\left(\frac{\beta_{2} \hat{Z}_{2}}{\alpha_{2}+\hat{P}}-\frac{\beta_{2} \hat{P} \hat{Z}}{\left(\alpha_{2}+\hat{P}\right)^{2}}\right) e^{-\lambda \tau_{2}}
\end{aligned}
$$




$$
b_{32}=0, \quad b_{33}=\lambda+d_{2}+2 g_{2} \hat{Z}_{2}+\frac{\rho_{2} \hat{P}}{\alpha_{2}+\hat{P}}-\frac{\beta_{2} \hat{P}}{\alpha_{2}+\hat{P}} e^{-\lambda \tau_{2}}
$$

The zeros of $\operatorname{det}\left(\Delta\left(\hat{u}, \tau_{2}, T\right)\right)(\lambda)=0$ are called characteristic roots. From $\left(A_{2}\right)$ we know that $\lambda=0$ is not a characteristic root. $\left(\hat{u}, \hat{\tau}_{2}, \hat{T}\right)$ is called a center if $\operatorname{det}\left(\Delta_{\hat{u}}\left(\operatorname{im} \frac{2 \pi}{\hat{T}}\right)\right)=0$. A center $\left(\hat{u}, \hat{\tau}_{2}, \hat{T}\right)$ is said to be isolated if it is the only center in some neighborhood of $\left(\hat{u}, \hat{\tau}_{2}, \hat{T}\right)$ and it has a finite characteristic value of form $\operatorname{im} \frac{2 \pi}{\hat{T}}$.

From Lemma 2.2 we conclude that $\left(u^{*}, \tau_{20}^{*(j)}, \frac{2 \pi}{\omega_{20}^{*}}\right), j=0,1,2, \ldots$, is an isolated center. There exists a smooth curve $\lambda:\left(\tau_{20}^{*(j)}-\delta, \tau_{20}^{*(j)}+\delta\right) \rightarrow C$ such that $\operatorname{det}\left(\Delta\left(u^{*}, \tau_{20}^{*(j)}\right.\right.$, $\left.\frac{2 \pi}{\omega_{20}^{*}}\right)\left(\lambda\left(\tau_{2}\right)\right)=0,\left|\lambda\left(\tau_{2}\right)-i \omega_{20}^{*}\right|<\varepsilon$ for $\tau_{2} \in\left(\tau_{20}^{*(j)}-\delta, \tau_{20}^{*(j)}+\delta\right), \lambda\left(\tau_{20}^{*(j)}\right)=i \omega_{20}^{*}$ and

$$
\left.\operatorname{Re} \frac{d \lambda}{d \tau_{2}}\right|_{\tau_{2}=\tau_{20}^{*(j)}}>0
$$

Define $\Omega_{\varepsilon, \frac{2 \pi}{\omega_{20}^{*}}}=\left\{(v, T): 0<v<\varepsilon,\left|T-\frac{2 \pi}{\omega_{20}^{*}}\right|<\varepsilon\right\}$.

$\left(A_{4}\right)$ If and only if $v=0, \tau_{2}=\tau_{20}^{*(j)}, T=\frac{2 \pi}{\omega_{20}^{*}}, j=0,1,2, \ldots, \operatorname{det}\left(\Delta\left(u^{*}, \tau_{2}, T\right)\left(v+i \frac{2 \pi}{T}\right)\right)=0$, hypotheses $\left(A_{1}\right)-\left(A_{4}\right)$ in [14] hold, we put $H^{ \pm}\left(u^{*}, \tau_{20}^{*(j)}, \frac{2 \pi}{\omega_{20}^{*}}\right)(v, T)=\operatorname{det}\left(\Delta\left(u^{*}, \tau_{20}^{*(j)} \pm\right.\right.$ $\left.\left.\delta, \frac{2 \pi}{\omega_{20}^{*}}\right)\left(v+\frac{2 \pi i}{T}\right)\right)$.

The crossing number $\gamma\left(u^{*}, \tau_{20}^{*(j)}, \frac{2 \pi}{\omega_{20}^{*}}\right)$ of center $\left(u^{*}, \tau_{20}^{*(j)}, \frac{2 \pi}{\omega_{20}^{*}}\right)$ can be defined as follows:

$$
\begin{aligned}
\gamma\left(u^{*}, \tau_{20}^{*(j)}, \frac{2 \pi}{\omega_{20}^{*}}\right)= & \operatorname{deg}_{B}\left(H^{-}\left(u^{*}, \tau_{20}^{*(j)}, \frac{2 \pi}{\omega_{20}^{*}}\right), \Omega_{\left(\varepsilon, \frac{2 \pi}{\omega_{20}^{*}}\right)}\right) \\
& -\operatorname{deg}_{B}\left(H^{+}\left(u^{*}, \tau_{20}^{*(j)}, \frac{2 \pi}{\omega_{20}^{*}}\right), \Omega_{\left(\varepsilon, \frac{2 \pi}{\omega_{20}^{*}}\right)}\right)=-1 .
\end{aligned}
$$

For the periodic solution of (1.2), we define $\Sigma(F)=C l\left(\left(u, \tau_{2}, T\right) \in X \times R_{+} \times R_{+} \mid u\right.$ is a T-periodic solution) $N(F)=\left(\left(\hat{u}, \hat{\tau_{2}}, \hat{T}\right) \in R_{+}^{5} \mid F\left(\hat{u}, \hat{\tau_{2}}, \hat{T}\right)=0\right)$.

Let $l\left(u^{*}, \tau_{20}^{*(j)}, \frac{2 \pi}{\omega_{20}^{*}}\right)$ denote the connected component of $\left(u^{*}, \tau_{20}^{*(j)}, \frac{2 \pi}{\omega_{20}^{*}}\right)$ in $\Sigma(F)$ for $\left(\hat{u}, \hat{\tau}_{2}\right.$, $\hat{T})=\left(u^{*}, \tau_{20}^{*(j)}, \frac{2 \pi}{\omega_{20}^{*}}\right)$, we obtain

$$
\Sigma_{\left(\hat{u}, \hat{\tau}_{2}, \hat{T}\right) \in l\left(u^{*}, \tau_{20}^{*(j)}, \frac{2 \pi}{\omega_{20}^{*}}\right) \cap N(F)} \gamma\left(\hat{u}, \hat{\tau}_{2}, \hat{T}\right)<0 .
$$

The connected component $l\left(u^{*}, \tau_{20}^{*(j)}, \frac{2 \pi}{\omega_{20}^{*}}\right)$ through $\left(u^{*}, \tau_{20}^{*(j)}, \frac{2 \pi}{\omega_{20}^{*}}\right)$ in $\Sigma(F)$ is not empty, since the first crossing number of each center is always -1 . By Theorem 3.3 [14], we conclude that $l\left(u^{*}, \tau_{20}^{*(j)}, \frac{2 \pi}{\omega_{20}^{*}}\right)$ is unbounded, which is presented as follows.

Lemma 3.1 $l\left(u^{*}, \tau_{20}^{*(j)}, \frac{2 \pi}{\omega_{20}^{*}}\right)$ is unbounded for each center $\left(u^{*}, \tau_{20}^{*(j)}, \frac{2 \pi}{\omega_{20}^{*}}\right)$.

By the fundamental theory [19], system (1.2) admits the existence and uniqueness of the solution with the initial condition

$$
\begin{aligned}
& P(\theta)=\phi(\theta) \geq 0, \\
& Z_{i}(\theta)=\psi_{i}(\theta) \geq 0, \quad \theta \in\left[-\tau_{2}, 0\right], \phi(0)>0, \psi_{i}(0)>0,(i=1,2) .
\end{aligned}
$$


Lemma 3.2 (Lemma 2.1 [1]) All the solutions of system (1.2) with initial condition (3.5) are positive and bounded on $[0,+\infty)$.

In [1], we have demonstrated this theorem, so we omit here.

Lemma 3.3 If system (1.2) has no nontrivial periodic solution with period $\tau_{2}$.

Proof: For system (1.2), suppose that there is a nontrivial periodic solution with period $\tau_{2}$, then the following system has a nontrivial periodic solution:

$$
\left\{\begin{array}{l}
\frac{d P}{d t}=r P\left(1-\frac{P}{K}\right)-\frac{\mu_{1} P Z_{1}}{\alpha_{1}+P}-\frac{\mu_{2} P Z_{2}}{\alpha_{2}+P}, \\
\frac{d Z_{1}}{d t}=\frac{\beta_{1} P\left(t-\tau_{1}\right) Z_{1}\left(t-\tau_{1}\right)}{\alpha_{1}+P\left(t-\tau_{1}\right)}-\frac{\rho_{1} P Z_{1}}{\alpha_{1}+P}-d_{1} Z_{1}-g_{1} Z_{1}^{2}, \\
\frac{d Z_{2}}{d t}=\frac{\beta_{2} P Z_{2}}{\alpha_{2}+P}-\frac{\rho_{2} P Z_{2}}{\alpha_{2}+P}-d_{2} Z_{2}-g_{2} Z_{2}^{2} .
\end{array}\right.
$$

We know that the $P-Z_{1}$-plane and the $Z_{1}-Z_{2}$-plane are invariant manifolds for system (3.6), there are no orbits crossing these two planes for the orbits do not intersect each other. Let $t^{\prime}=\inf \left\{t: Z_{1}(t)=0\right\}$, combining with initial condition (3.5) and the second equation of (3.6), we get

$$
\left.\frac{d Z_{1}}{d t}\right|_{t=t^{\prime}}=\frac{\beta_{1} P\left(t^{\prime}-\tau_{1}\right) Z_{1}\left(t^{\prime}-\tau_{1}\right)}{\alpha_{1}+P\left(t^{\prime}-\tau_{1}\right)} \geq 0
$$

from which the orbits do not cross the $P-Z_{2}$-plane, so all the orbits must lie in the first quadrant and equilibrium $E^{*}$ should be in the interior of a periodic solution. From Lemma $2.1\left(\tau_{1} \in\left(0, \tau_{10}\right), \tau_{2}=0\right), E^{*}$ is stable and unique, so it is globally stable in the first quadrant, the periodic solution does not exist. Thus there is no periodic orbit in the first quadrant.

Theorem 3.1 For each $\tau_{2}>\tau_{20}^{*(j)}, j=0,1,2, \ldots$, system $(1.2)$ has at least $j+1$ periodic solutions.

Proof It is sufficient to prove that the connected component $l\left(u^{*}, \tau_{20}^{*(j)}, \frac{2 \pi}{\omega_{20}^{*}}\right)$ onto the $\tau_{2}$ space is $(\bar{\tau},+\infty)$, where $\bar{\tau} \leq \tau_{20}^{*(j)}, j=0,1,2, \ldots$. From Lemma 3.3 , we know that system (3.6) does not have a nontrivial periodic solution, so the projection of $l\left(u^{*}, \tau_{20}^{*(j)}, \frac{2 \pi}{\omega_{20}^{*}}\right)$ onto the $\tau_{2}$ space is always from zeros. From expression (2.2), we get

$$
\tau_{20}^{*(j)}=\frac{1}{\omega_{20}^{*}} \operatorname{arcos}\left(\frac{R_{52} R_{53}-R_{51} R_{54}}{R_{51}^{2}+R_{52}^{2}}\right)+\frac{2 j \pi}{\omega_{20}^{*}},
$$

so we obtain $\frac{2 \pi}{\omega_{20}^{*}}<\tau_{20}^{*(j)}$ for $j>0$. Suppose that the projection of $l\left(u^{*}, \tau_{20}^{*(j)}, \frac{2 \pi}{\omega_{20}^{*}}\right)$ onto the $\tau_{2}$ space is bounded, there exists $\tau^{*}>0$, so the projection of $l\left(u^{*}, \tau_{20}^{*(j)}, \frac{2 \pi}{\omega_{20}^{*}}\right)$ onto the $\tau_{2}$ space is in the interval $\left(0, \tau^{*}\right)$. From $\frac{2 \pi}{\omega_{20}^{*}}<\tau_{20}^{*(j)}(j \geq 1)$ and Lemma 3.3, $0<T<$ $\tau^{*}$ for $\left(u(t), \tau_{2}, T\right) \in l\left(u^{*}, \tau_{20}^{*(j)}, \frac{2 \pi}{\omega_{20}^{*}}\right)$, which means that the projection of $l\left(u^{*}, \tau_{20}^{*(j)}, \frac{2 \pi}{\omega_{20}^{*}}\right)$ onto the $T$ space is also bounded. By Lemma 3.2, we obtain that the connected com- 

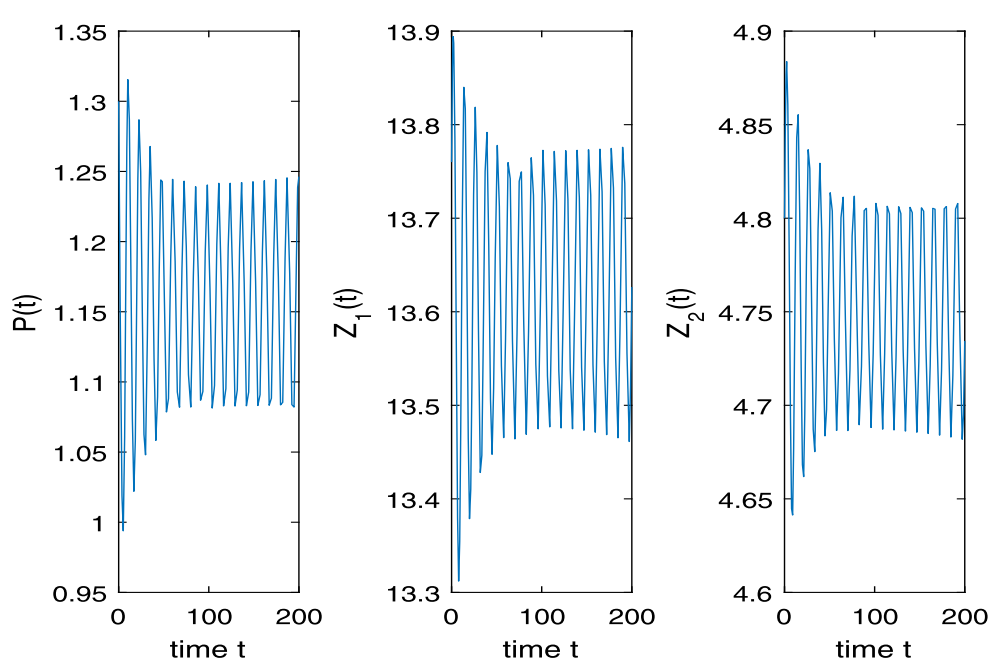

Figure 1 When $\tau_{1}=0.2, \tau_{2}=0.78853$, the periodic solution bifurcates from $E^{*}$

ponent $l\left(u^{*}, \tau_{20}^{*(j)}, \frac{2 \pi}{\omega_{20}^{*}}\right)$ is bounded, which contradicts Lemma 3.1, so the proof is completed.

\section{Numerical simulation}

We give some simulation to support our result. We take the same parameter to [1], system (1.2) becomes

$$
\left\{\begin{array}{l}
\frac{d P}{d t}=5 P\left(1-\frac{P}{4}\right)-\frac{0.6 P Z_{1}}{2+P}-\frac{0.64 P Z_{1}}{2+P}, \\
\frac{d Z_{1}}{d t}=\frac{0.43 P\left(t-\tau_{1}\right) Z_{1}\left(t-\tau_{1}\right)}{2+P\left(t-\tau_{1}\right)}-\frac{0.1 P Z_{1}}{2+P}-0.11 Z_{1}-0.0008 Z_{1}^{2}, \\
\frac{d Z_{2}}{d t}=\frac{0.53 P\left(t-\tau_{2}\right) Z_{2}\left(t-\tau_{2}\right)}{2+P\left(t-\tau_{2}\right)}-\frac{0.1 P Z_{2}}{2+P}-0.11 Z_{2}-0.01 Z_{2}^{2} .
\end{array}\right.
$$

From [1] we know that system (4.1) has a unique equilibrium $E^{*}=(1.15632,13.62813$, 4.75297). For case (2), $\tau_{10}=0.79432$. For case (5), fixing $\tau_{1}=0.2$, then $\tau_{20}^{*}=0.32688$, when $\tau_{2}=0.30115<\tau_{20}^{*}, E^{*}$ is asymptotically stable, when $\tau_{2}=0.94733>\tau_{20}^{*}$, Hopf bifurcation occurs and a periodic solution bifurcates from $E^{*}$, which is illustrated in Figs. 7 and 8 in [1].

Furthermore, we know that system (1.2) has a periodic solution for large $\tau_{2},\left(\tau_{1} \in\left(0, \tau_{10}\right)\right)$ from global Hopf bifurcation. Figures 1, 2, and 3 show the amplitude and period of a bifurcating periodic solution for different values of $\tau_{2}\left(\tau_{2}=0.78853,2.20041,5.75432\right)$. We conclude that the amplitude and period increase as $\tau_{2}$ increases.

\section{Conclusion}

In this paper, we study the global Hopf bifurcation of two zooplankton-phytoplankton system with two delays. Fixing the delay $\tau_{1}$ in its stable interval and taking the delay $\tau_{2}$ as a parameter, we demonstrate that the bifurcating periodic solution exists as $\tau_{2}$ increases, where the delays $\tau_{1}, \tau_{2}$ are due to gestation of two zooplankton. We conclude the global oscillatory nature for the solution of a delayed system when it exhibits oscillatory behavior. Our work answers the question in Sect. 5 [1]. 

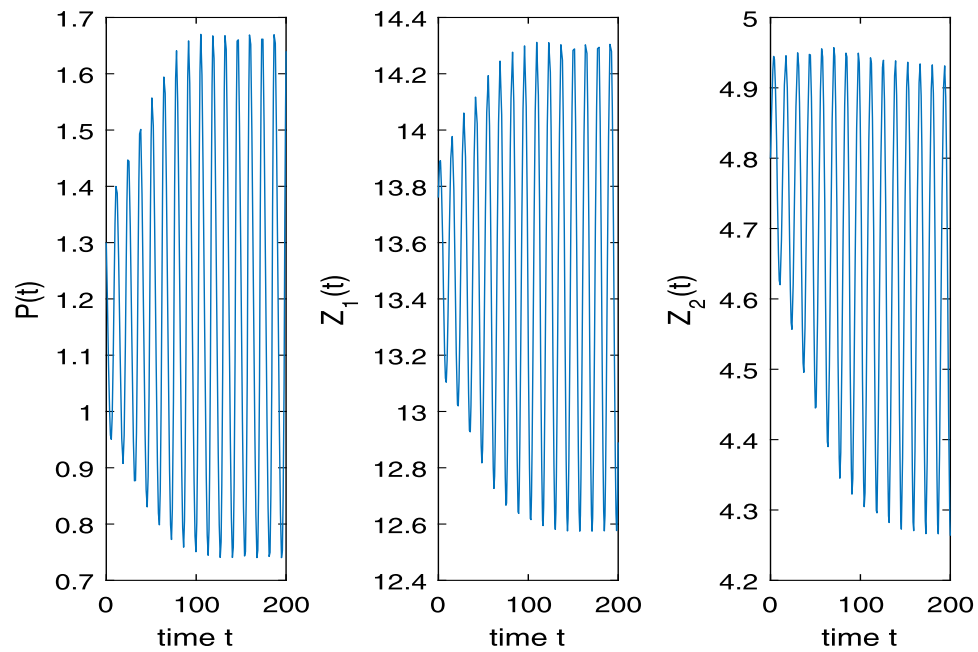

Figure 2 When $\tau_{1}=0.2, \tau_{2}=2.20041$, the periodic solution bifurcates from $E^{*}$
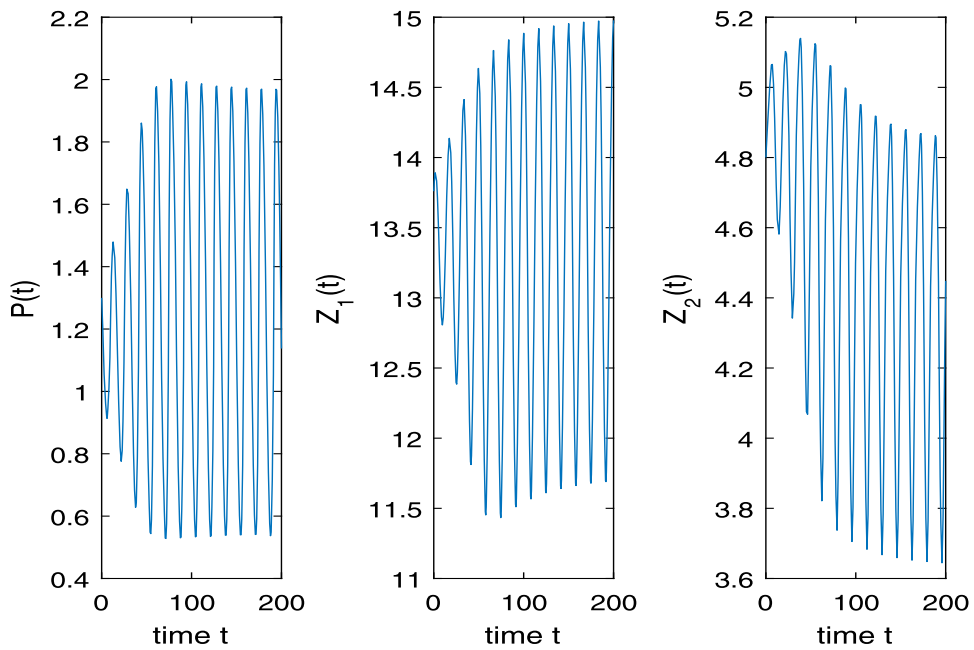

Figure 3 When $\tau_{1}=0.2, \tau_{2}=5.75432$, the periodic solution bifurcates from $E^{*}$

\section{Acknowledgements}

The authors express their sincere gratitude to the editors and referees for their helpful comments and suggestions.

\section{Funding}

This paper was supported by the National Natural Science Foundation of China (No. 11701292).

\section{Availability of data and materials}

The data and material come from papers [1, 4]. We think these data and material satisfy the condition which turns up in the paper. So we think the data and material are available.

\section{Competing interests}

The authors declare that they have no competing interests.

\section{Authors' contributions}

The main idea of this paper was proposed by RS and WY. RS prepared the manuscript initially and performed all the steps of the proofs in this research. All authors read and approved the final manuscript. 


\section{Publisher's Note}

Springer Nature remains neutral with regard to jurisdictional claims in published maps and institutional affiliations.

Received: 19 May 2020 Accepted: 15 October 2020 Published online: 27 October 2020

\section{References}

1. Shi, R., Yu, J.: Hopf bifurcation analysis of two zooplankton-phytoplankton model with two delays. Chaos Solitons Fractals 100, 62-73 (2017)

2. Sharma, A., Sharma, A., Agnihotri, K.: Analysis of a toxin producing phytoplankton-zooplankton interaction with Holling IV type scheme and time delay. Nonlinear Dyn. 81, 13-25 (2015)

3. Das, K., Ray, S.: Effect of delay on nutrient cycling in phytoplankton-zooplankton interactions in estuarine system. Ecol. Model. 215, 69-76 (2008)

4. LV, Y., Cao, J., Song, J., Yuan, R., Pei, Y.: Global stability and Hopf-bifurcation in a zooplankton-phytoplankton model. Nonlinear Dyn. 76, 345-366 (2014)

5. Wang, Y., Wang, H., Jiang, W.: Stability switches and global Hopf bifurcation in a nutrient-plankton model. Nonlinear Dyn. 78, 981-994 (2014)

6. Zhao, J., Wei, J.: Stability and bifurcation in a two harmful phytoplankton-zooplankton system. Chaos Solitons Fractals 39, 1395-1409 (2009)

7. Wang, Y., Jiang, W., Wang, H.: Stability and global Hopf bifurcation in toxic phytoplankton-zooplankton model with delay and selective harvesting. Nonlinear Dyn. 73, 881-896 (2013)

8. Saha, T., Bandyopadhyay, M.: Dynamical analysis of toxic producing phytoplankton-zooplankton interactions. Nonlinear Anal., Real World Appl. 10, 314-332 (2009)

9. Gakkhar, S., Singh, A.: Effects of delay and seasonality on toxin producing phytoplankton-zooplankton system. Int. J. Biomath. 5, 1250047 (2012)

10. Shi, R., Yu, J.: Global Hopf bifurcation in a delayed phytoplankton-zooplankton model with competition. Ann. Appl. Math. 33(1), 32-49 (2017)

11. Zeng, X., Xiong, Z., Wang, C.: Hopf bifurcation for neutral-type neural network model with two model with delays. Appl. Math. Comput. 282, 17-31 (2016)

12. Liu, J., Sun, L.: Dynamical analysis of a food chain system with two delays. Qual. Theory Dyn. Syst. 15, 95-126 (2016)

13. Deng, L., Wang, X., Peng, M.: Hopf bifurcation analysis for a ratio-dependent predator-prey system with two delays and stage structure for the predator. Appl. Math. Comput. 231, 214-230 (2014)

14. Wu, J.: Symmetric functional differential equations and neural networks with memory. Trans. Am. Math. Soc. 350 , 4799-4838 (1998)

15. Meng, X., Huo, H., Zhang, X.: Stability and global Hopf bifurcation in a delayed food web consisting of a prey and two predators. Commun. Nonlinear Sci. Numer. Simul. 16, 4335-4348 (2011)

16. Xu, J., Zhou, Y.: Hopf bifurcation and its stability for a vector-borne disease model with delay and reinfection. Appl. Math. Model. 40, 1685-1702 (2016)

17. Li, X.-L.: Global existence of periodic solutions in a physiological model with delay. Acta Math. Appl. Sin. Engl. Ser. 31, 1043-1048 (2015)

18. Hassard, B., Kazarinoff, N.: Wan, Y. Theory and Application of Hopf Bifurcation. Cambridge University Press, Cambridge (1981)

19. Hale, J., Lunel, S.: Introduction to Functional Differential Equation. Springer, New York (1993)

\section{Submit your manuscript to a SpringerOpen ${ }^{\circ}$ journal and benefit from:}

- Convenient online submission

- Rigorous peer review

- Open access: articles freely available online

- High visibility within the field

- Retaining the copyright to your article

Submit your next manuscript at $\downarrow$ springeropen.com 